\title{
An In Vivo Electrophysiological Method for Measuring Olfactory Dysfunction
}

\author{
Liujing Zhuang1, Bin Zhang1, Zhen Qin'1, Xinyi Lai², Ping Wang ${ }^{1}$ \\ ${ }^{1}$ Biosensor National Special Laboratory, Key Laboratory for Biomedical Engineering of Education \\ Ministry, Department of Biomedical Engineering, Zhejiang University, Hangzhou 310027, China \\ 2 Interdisciplinary Institute of Neuroscience and Technology, Qiushi Academy for Advanced Studies, \\ Zhejiang University, Hangzhou, China \\ cnpwang@zju.edu.cn
}

\begin{abstract}
:
Olfaction provides important functions and olfactory dysfunction (OD) impair quality of life. Here we use implantable microelectrode array to extract the spontaneous neural activity of olfactory system. By analyzing the correspondence between neural activity and olfactory function, we extract the feature signals which could represent the olfactory function, and decode the feature signals into measurement parameters. According to the quantitative description of olfactory function, we can develop a olfactory model for clinically simple, fast and reliable diagnosis of OD.
\end{abstract}

Key words: Olfactory function measurement, implantable microelectrode, electrophysiological recording, spontaneous neural activity, neural decoding

\section{Introduction}

Olfaction plays an important role in quality of human life. Olfactory dysfunction (OD) influences food selection and nutrient intake, and compromises safety from food poisoning and toxic agents [1]. It can be devastating for those who depend upon olfaction for their safety or livelihood, such as homemakers, cooks, plumbers, fire fighters, perfumers and so on. In recent years, psychophysical and electrophysiological tests have been developed to quantify olfactory function in the clinical setting. However, as soon as the patient's collaboration is not guaranteed, interpretation of psychophysical test results becomes difficult or even impossible [2]. And electrophysiological recording is not reliable and repeatable. Therefore, diagnostic method for OD is still in a state of unmet need [3].

In mammalian olfactory system, the initial event of olfactory sensing takes place when the odorant molecules are bound to the receptors of sensory neurons in the olfactory epithelium. Then the sensory neurons send olfactory information through their axon to the olfactory bulb (OB) for coding and processing before transmitting to the olfactory cortex [4].
Olfactory function is maintained by normal neural activity, we hypothesized that OD is accompanied by disrupted neural activity. To setup an effective method for OD diagnosis, we extracted and decoded neural activity in the olfactory bulb (OB) of animal models using implantable microelectrode array (MEA) probe. Characteristics of neural oscillations in normal and olfactory-impaired animals were presented here.

\section{Experimental method}

SOI based MEA probe was designed and constructed by MEMS fabricating technology (see Fig. 1). The probe was chronically implanted in the mouse olfactory bulb through small craniotomy. Neural activities in freely behaving mouse were recorded. Two C57/BL6 mouse models with OD were created: in type 1 , one of the nasal cavities was occluded by a nose plug; in type 2 , the olfactory epithelium was damaged by zinc sulfate.

\section{Results}

(1) In the OB of mice with normal olfaction, respiration-locked oscillations are prominent and gamma oscillations $(60-100 \mathrm{~Hz})$ are phaseamplitude coupled to respiration-locked oscillation (see Fig. 2b left). (2) Respiration- 
locked oscillations are significantly reduced in OD mice with, and the power of gamma oscillation also dramatically decreased (see Fig. 2b). (3) The coupling strength between respiration rhythm phase and gamma amplitude dramatically decreases in OD mice (see Fig. $3 c)$.

\section{Discussion}

The coupling pattern between respirationlocked and gamma oscillation is significant
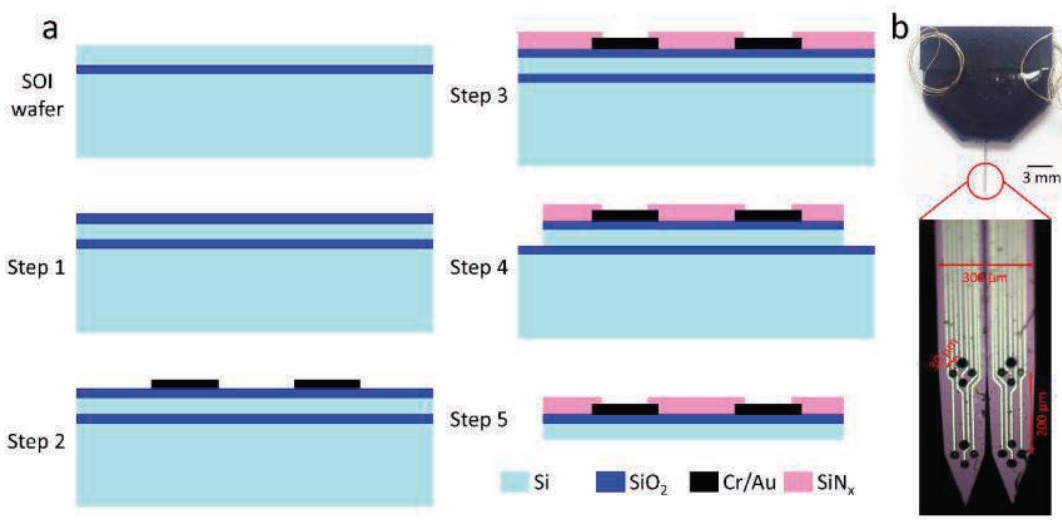

Fig. 1 (a) Schematic of the MEA probe fabrication process. (b) Encapsulated MEA probe (top) and photomicrograph of Pt black modified probe tip (bottom).
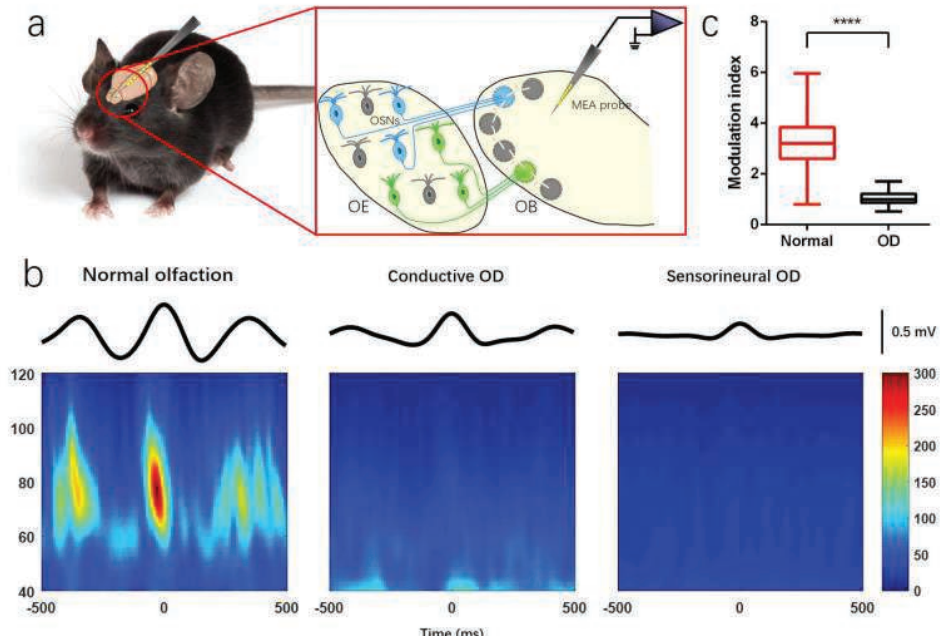

Fig. 2 (a) Schematic of in vivo neural recording from mouse olfactory bulb (OB). (b) Mean time-frequency powerspectrum of gamma oscillation (40-120 Hz, bottom) and respiration rhythm (1-5 Hz, top) peak-locked mean raw signal in control (left), naris occluded (middle), olfactory epithelium (right) mouse. (c) Modulation index was computed to evaluate the strength of coupling between respiration rhythm phase and gamma amplitude in normal and $O B$ mice $\left(n=8\right.$, unpaired two-tailed t test, $\left.{ }^{* * *} P<0.0001\right)$.

\section{References}

[1] R. L. Doty, Olfactory dysfunction and its measurement in the clinic. World journal of otorhinolaryngology-head and neck surgery 1 , 28-33 (2015); doi: 10.1016/j.wjorl.2015.09.007

[2] A. Eibenstein, A. Fioretti, C. Lena, N. Rosati, G. Amabile, M. Fusetti, Modern psychophysical tests to assess olfactory function. Neurological Sciences 26, 147-155 (2005). doi:10.1007/s10072-005-0452-3
[3] J. Lötsch, T. Hummel, The clinical significance of electrophysiological measures of olfactory function. Behavioural brain research 170, 78-83 (2006). doi:10.1016/j.bbr.2006.02.013

[4] P. M. Lledo, G. Gheusi, J. D. Vincent, Information processing in the mammalian olfactory system. Physiological Reviews 85, 281-317 (2005); doi:10.1152/physrev.00008.2004 